\title{
Effectiveness of martial arts exercise on anthropometric and body composition parameters of overweight and obese subjects: a systematic review and meta- analysis
}

Fabricio de Souza ${ }^{1 *}$ (D), Felipe Nunes Lanzendorf², Márcia Mendonça Marcos de Souza ${ }^{3}$, Fabiana Schuelter-Trevisol ${ }^{1,4}$ and Daisson José Trevisol ${ }^{1,4}$

\begin{abstract}
Background: Obesity is considered a top public health concern, and its prevalence is growing every day. Thus, interventions to address this problem should be encouraged and further studied. In this regard, the aim of this review was to summarize the evidence of martial arts interventions to evaluate their effectiveness on the anthropometric and body composition parameters of overweight and obese subjects.

Methods: A systematic literature search was conducted on January 26, 2020 using the PubMed, Medline, Lilacs, Cochrane, and Scielo databases. Reference lists of eligible articles and relevant reviews have also been examined. All randomized clinical trials on martial arts that evaluated the anthropometric and body composition parameters of overweight and obese subjects were included, and a narrative synthesis of eligible studies was conducted in accordance with PRISMA guidelines. The Downs \& Black checklist was used to assess the quality of the studies. This review was registered in the International Prospective Register of Systematic Reviews (PROSPERO) (identifier CRD42018086116).

Results: A total of 82 articles were identified from the initial search strategy. A further 2 articles were identified from the review of relevant bibliographies. Six studies encompassing 258 participants who were overweight or obese were included. Four studies reported Tai Chi practice, one study reported Kung Fu exercise, and another study reported martial arts exercise. The examined meta-analyses did not reveal significant benefits from martial arts practice over control groups after the experiment period for body mass index $\left(-1.34 \mathrm{~kg} / \mathrm{m}^{2} ; 95 \% \mathrm{Cl}:-2.72,0.05\right)$, waist circumference $(1.41 \mathrm{~cm} ; 95 \% \mathrm{Cl}$ : $-0.72,3.54)$ and percentage of body fat $(-0.75 \% ; 95 \% \mathrm{Cl}:-5.58,4.08)$.
\end{abstract}

(Continued on next page)

\footnotetext{
* Correspondence: fabriciokarate@hotmail.com

'Postgraduate Program in Health Sciences, University of Southern Santa Catarina, Avenida José Acácio Moreira, 787, Bairro Dehon, Tubarão, Santa Catarina Caixa Postal 370, Brazil

Full list of author information is available at the end of the article
}

(c) The Author(s). 2020 Open Access This article is licensed under a Creative Commons Attribution 4.0 International License, which permits use, sharing, adaptation, distribution and reproduction in any medium or format, as long as you give appropriate credit to the original author(s) and the source, provide a link to the Creative Commons licence, and indicate if changes were made. The images or other third party material in this article are included in the article's Creative Commons licence, unless indicated otherwise in a credit line to the material. If material is not included in the article's Creative Commons licence and your intended use is not permitted by statutory regulation or exceeds the permitted use, you will need to obtain permission directly from the copyright holder. To view a copy of this licence, visit http://creativecommons.org/licenses/by/4.0/ The Creative Commons Public Domain Dedication waiver (http://creativecommons.org/publicdomain/zero/1.0/) applies to the data made available in this article, unless otherwise stated in a credit line to the data. 
(Continued from previous page)

Conclusion: The scarcity, heterogeneity, short intervention time, small sample size, and significant methodological limitations of the available studies do not allow to conclude whether martial arts are effective in the anthropometric and body composition parameters of overweight and obese individuals. This study highlights the need for more research to assess the benefits of martial arts for overweight and obese individuals.

Keywords: Body mass index, Weight loss, Exercise, Physical activity

\section{Background}

Obesity is a chronic disorder of multifactorial origin, defined as an accumulation of abnormal or excessive fat that can be detrimental to health [1]. Currently, obesity is one of the most serious public health problems worldwide, being more prevalent than malnutrition itself [2]. According to the World Health Organization (WHO) [1], more than 1.9 billion adults were overweight, of whom more than 650 million were obese in 2016. These figures indicated that 39\% of the adult world population was overweight, and $13 \%$ were obese [1]. In 2016, the WHO also estimated that 41 million children under the age of 5 , and 340 million children and adolescents aged 5-19 years were overweight or obese worldwide [1].

Lifestyle interventions that provide a $5-10 \%$ reduction in body weight are recommended as a strategy to combat obesity [3, 4]. However, excess adipose tissue, especially visceral adipose tissue, poses greater risks to health than excess body weight [5]. Therefore, interventions aiming at weight loss should seek changes in anthropometric parameters and body composition, since the reduction of visceral adipose tissue decreases cardiovascular risks and metabolic issues [6].

Interventions for treating overweight and obese people include nutritional education and changes in lifestyle, which require increasing levels of physical activity and the reduction of sedentary behaviors [7]. The increase in energy expenditure through regular physical exercise is an interesting alternative for the treatment of overweight and obese people. Besides being a simple option to help control body weight [1], physical exercise can also produce positive health effects, such as cardiorespiratory fitness, reduction of inflammatory markers, elevation of anti-inflammatory markers, and improvements in cardiometabolic risk factors [8]. Studies have shown that aerobic exercise is the main form of exercise for obesity prevention and control [9-14]. However, some studies have reported important results from different treatment interventions for overweight and obese subjects, mainly interventions that used different intensity levels and the combination of aerobic exercises with strength exercises [15-18]. Thus, studies that investigate other forms of intervention with the use of physical exercise should be taken into account [15].
An organized activity that emerges as an alternative form of physical activity and that is gaining many supporters is the practice of martial arts [19-21]. Martial arts were developed for use on the battlefield [20, 21]. However, in the current context, they are used for a variety of purposes, including fitness, sports, self-defense, combat skills, meditation, character development, selfconfidence, and health-related aspects [19-21]. In addition, they can be used as complementary or alternative therapy for some medical conditions [22]. Martial arts practitioners find great pleasure in performing physical activity, which is an extremely important factor, because in addition to encouraging participation, it contributes to better treatment results [23].

Despite the studies reporting and analyzing the benefits of practicing martial arts in the general population, scientific evidence regarding their use for the treatment of overweight and obese subjects is limited. Therefore, before recommending martial arts as a treatment for weight loss, it is important to analyze the scientific evidence for potential benefits and risks. For that reason, the purpose of this systematic review was to evaluate the effectiveness of martial arts exercise on the anthropometric and body composition parameters of overweight and obese subjects.

\section{Methods}

The PRISMA Statement (Preferred Reporting Items for Systematic reviews and Meta-Analyses) [24] was used to conduct and report the systematic review. This review was registered in the International Prospective Register of Systematic Reviews (PROSPERO) (identifier CRD42018086116). However, no study protocol was published in a journal before.

\section{Eligibility criteria}

The inclusion criteria were the following: (1) randomized controlled trials study design; (2) published in English, Spanish or Portuguese; (3) martial arts exercise, isolated or combined with any other practices; (4) overweight or obese participants (body mass index $[\mathrm{BMI}] \geq 85$ percentile for children and adolescents, and BMI > 25 for adults); (5) use of a quantitative method to determine at least one of the relevant measurements of anthropometric and body 
composition parameters (BMI, body-fat percentage, or waist circumference).

\section{Search strategy}

To ensure the inclusion of relevant literature, the electronic search was conducted in different databases, namely PubMed, Medline, Lilacs, Cochrane, and Scielo in January 26, 2020. The adopted search strategy included a combination of the following Medical Subject Headings (MeSH) terms: obesity AND martial arts. Keyword iterations related to these terms were also used. Example of the search strategy in PubMed: Obesity [all fields]; Obes* [all fields]; Overweight [all fields]; \#1 OR \#2 OR \#3; Martial arts [all fields]; Martial Fitness [all fields]; Martial exerc* [all fields]; \#5 OR \#6 OR \#7; \#4 AND \#8 (supplementary file 1). Also, the reference lists of the included studies were examined as an additional check for potential studies that could be used in this review.

\section{Selection of studies and data extraction}

Initially, all titles and abstracts were screened independently by two researchers (F.S. and F.N.L). Each researcher selected the studies that met the inclusion criteria. Full texts were read by 2 authors (FS, FNL) and the final list of eligible studies was compiled. If discrepancies between the 2 researchers occurred those items were discussed with a third researcher (DJT) to achieve a consensus. After selecting the articles, the following data were extracted: author, date of publication, language, sample size, demographic characteristics (Country/setting, age and ethnicity), BMI, body composition, intervention and control, and outcomes. The researchers were not blinded to the journal titles and paper authors for the data extraction process.

\section{Bias risk assessment}

The methodological quality of the studies was evaluated independently by two researchers (FS and FNL), using the Downs \& Black checklist [25]. This assessment tool has 27 scoring items to assess the strength of reporting, external validity, internal validity, and statistical power of randomized and non-randomized studies of health care interventions (Additional file 2). In that tool, the maximum score that can be received is 32 . The score obtained by each study was divided by 32 and multiplied by 100 to provide a 'Study Quality Percentage', considering the following breakdown: high quality $(66.7 \%$ or more); medium quality (between 50.0-66.6\%), and low quality (less than 50.0\%) [26]. Doubts and divergences between the researchers were resolved through consensus and mediated by a third researcher (DJT).

\section{Data analysis}

The systematic review data were analyzed and presented employing a narrative synthesis approach. We examined the year of publication, country of origin, study design, sample characteristics, types of martial arts selected for intervention, outcome measures and treatments applied to the control groups. The overall effect sizes for each outcome were determined using meta-analytic approaches if at least 2 studies assessed the specific outcome. The Review Manager 5 software (Version 5.4, The Nordic Cochrane Centre Copenhagen) was used, and random effects models were applied. Meta-analyses were conducted to examine the effects of martial arts compared with control groups. Mean differences (MD) with 95\% confidence intervals (CI) were reported. Attempts were made to obtain missing data from the studies' authors by email.

Statistical heterogeneity between the studies was determined using $\mathrm{I}^{2}$ statistics, and the magnitude of heterogeneity was categorized as $\mathrm{I}^{2}=0$ to $24 \%$ : low; $\mathrm{I}^{2}=25$ to 49\%: moderate; $\mathrm{I}^{2}=50$ to $74 \%$ : substantial; and $\mathrm{I}^{2}=75$ to $100 \%$ : considerable heterogeneity $[27,28]$; and the $x^{2}$ test was used to assess the statistical significance of heterogeneity between trials. In the face of the low power of this test in small samples, a $P$ value $\leq 0.10$ was regarded to indicate significant heterogeneity [29].

\section{Results}

The electronic search initially retrieved 82 titles. Two additional articles were found in the reference section of the review articles. After duplicate articles were removed, 66 articles were screened by reading the titles and abstracts. Of them, 52 articles were excluded because they did not meet the inclusion criteria. The remaining 14 articles were selected for further review. After full reading, 8 studies were excluded (3 did not address overweight or obese subjects and 5 did not evaluate body composition). The study selection process is shown in the PRISMA [24] flow diagram (Fig. 1). A total of 6 studies were included in this systematic review (Table 1).

\section{Characteristics of the studies}

Six randomized clinical trials $(n=6)$ [30-35] were included in this systematic review (Table 1). The selected studies were published between 2009 and 2013. Three of them were conducted in the United States (50\%) [31, 33, 35], one in Australia (16.66\%) [30], one in France (16.66\%) [34], and one in China (16.66\%) [32]. The analysis encompassed 258 overweight or obese subjects. Sample sizes ranged from 20 to 117, with a mean of 43 $(\mathrm{SD}=37.55)$. The majority of the participants were women $72.5 \%(n=187)$, with a mean age of 46.5 years $(\mathrm{SD}=18.66)$. BMI was taken as a criterion in all studies 


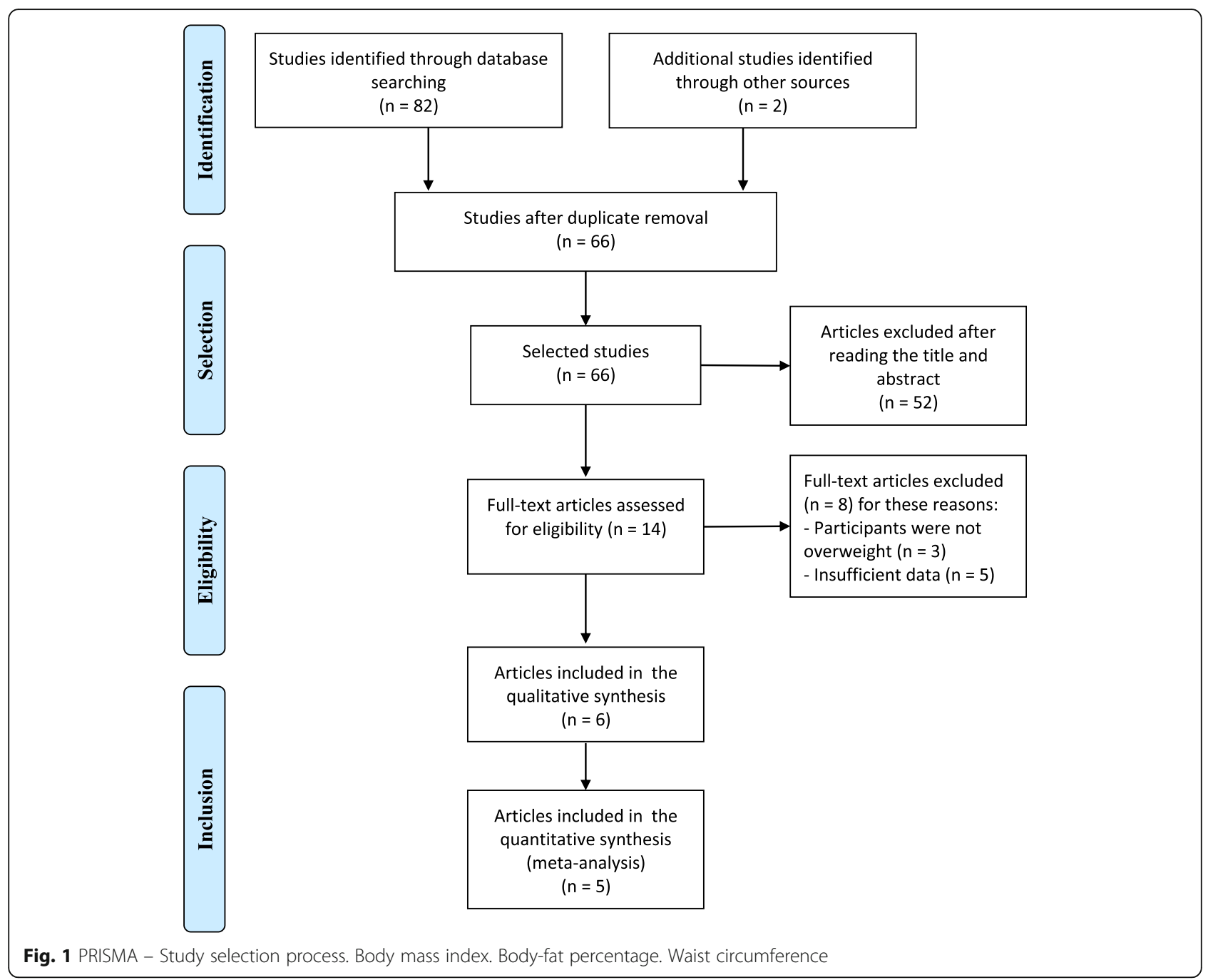

for identifying the overweight or obese sample 100\% $(n=6)$, but different criteria were adopted in various studies to define overweight and obesity. Most studies $(66.66 \%)$ defined a BMI $\geq 30(n=4)$ as a criterion for inclusion, one study (16.66\%) defined a $B M I \geq 25$, and one study $(16.66 \%)$ defined a $B M I \geq 85$ th percentile.

\section{Interventions}

Regarding the type of intervention, four studies (66.66\%) reported Tai Chi practice as an intervention technique [31, 32, 34, 35], one study (16.66\%) reported Kung Fu practice [30] and another study (16.66\%) reported different martial arts styles [33]. Number of intervention sessions per week ranged from 1 to 3 , with a mean of $2.66(\mathrm{SD}=$ 0.82 ). The duration in weeks ranged from 10 to 24 , with a mean of $15(\mathrm{SD}=5.02)$. It should be mentioned that no study used the same intervention methodology, differing between them in relation to the proposed interventions, frequency and duration of the sessions, additional interventions, and treatments in the control groups.
Tai Chi was the most common martial arts training applied as intervention in the treatment group 66.66\% $(n=4)$. The Yang style 24-posture Form was the most widely studied $75 \%(n=3)$ [31, 34, 35], and only one study by Chen and colleagues [32] reported the Chen style 99-posture form Tai Chi as the sole intervention in the treatment group. Three studies (75\%) [31, 34, 35] reported additional interventions besides Tai Chi training in the treatment group. Beebe and colleagues [31] adopted nutrition education as an additional intervention technique. Dechamps and colleagues [34] developed psychological and medical interventions, besides nutritional education, and diet. Katkowski and colleagues [35] employed nutritional interventions composed of lectures and diets.

Different interventions were performed in the control groups applying Tai Chi as the main intervention in the treatment group [31, 32, 34, 35]. Beebe and colleagues [31] used nutrition education as intervention in the control group. Two studies $(50 \%)[32,34]$ reported conventional training as intervention in the control group, but 


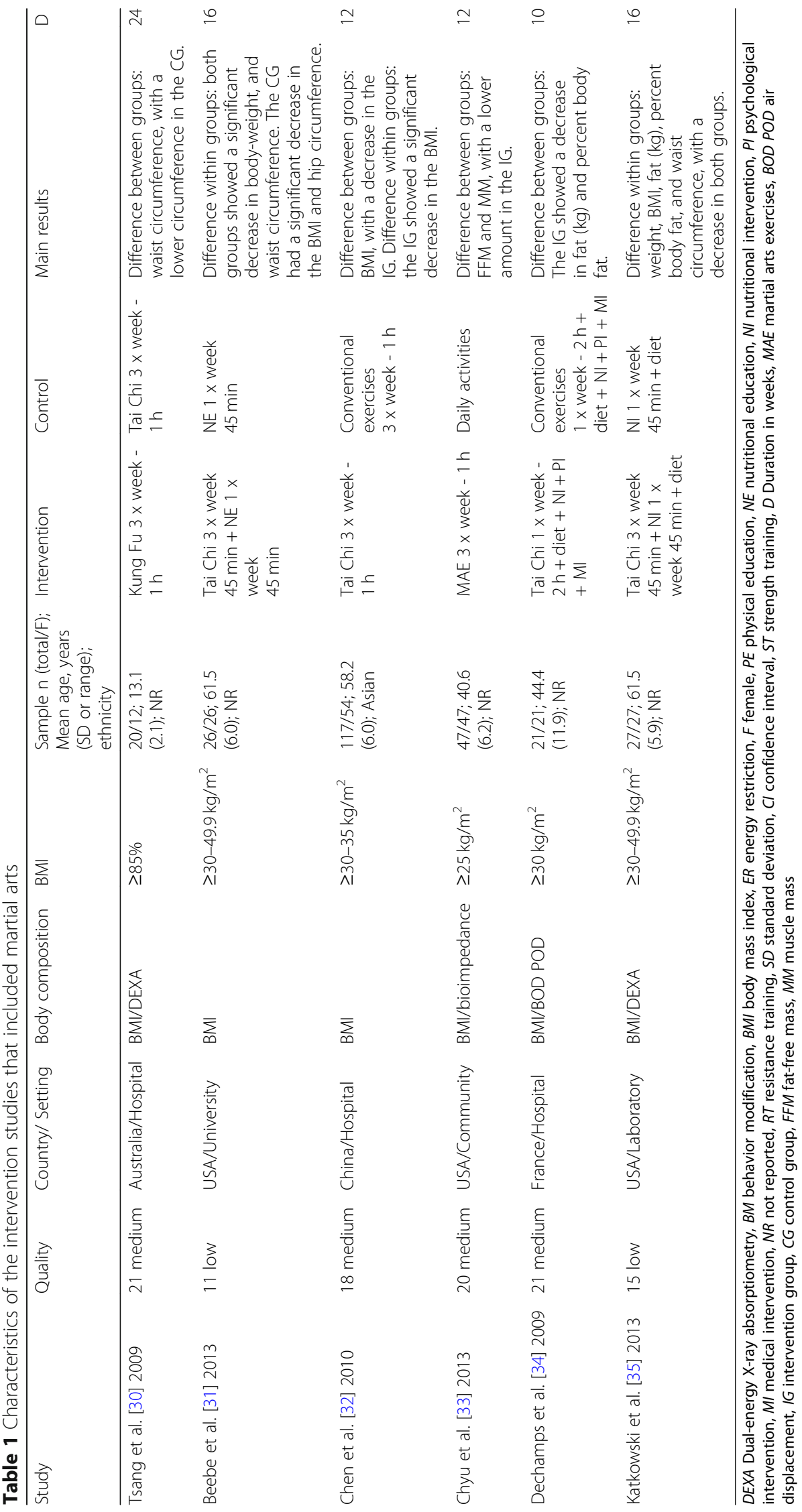


the study by Dechamps and collaborators [34] also adopted nutritional, psychological, and medical intervention, in addition to conventional training. Katkowski and colleagues [35] performed nutritional interventions composed of lectures and diets.

Two studies (33.33\%) [30,33] developed other forms of martial arts. Tsang and colleagues [30] investigated the effects the Kung Fu Choy Lee Fut Hung Sing Gwoon style as intervention and the Tai Chi Yang style 24posture form as placebo for the control group. Chyu and colleagues [33] studied martial arts exercise composed of traditional techniques of different martial arts styles as intervention in their study and recommended that the participants in the control group should maintain their daily activities.

\section{Participation}

Three studies have not reported the participation rate in their interventions [31, 32, 34]. Tsang and colleagues [30] reported that the participation rate in the interventions was less than 50\%. Chyu and colleagues [33] reported participation rates of $83.4 \%$. Katkowski and colleagues [35] subdivided the participation rate because of their multiple interventions. They reported that the participation rate of the martial arts intervention group was $73.1 \%$ for the Tai Chi technique and $78.1 \%$ for the dietary intervention. The participation rate in the control group was $86.5 \%$ for the dietary intervention.

\section{Adverse events}

Two studies [31, 35], which accounted for $33.33 \%$ of the studies included in this systematic review, did not report adverse events in their interventions. Dechamps and colleagues [34] reported the absence of adverse events in their study. Chen and colleagues [32] reported there were very few adverse events or lesions. Chyu and colleagues [33] reported the occurrence of muscular pain, knee pain, and shoulder pain in the first 2 weeks of intervention. Tsang and colleagues [30] reported two falls that occurred during the intervention period, noting that wearing inappropriate clothes was the cause of one of the falls.

\section{Quality of studies}

A quality index was assigned to each of the studies included in this systematic review. The score ranged from 11 to 21 , with a mean of $17.66(\mathrm{SD}=3.98)$ [25], as shown in Table 2 and Additional file 2. Quality rating indicated that $66.66 \%$ of the studies were rated medium quality $(n=4)$, and $33.33 \%$ were rated low quality $(n=2)$; none was rated high quality. Assessment of study quality in this systematic review revealed the following negative factors: no identification of the main confounding factors, lack of treatment control and details of adverse events, poor analysis of the participants lost in the follow-up, absence of random sampling to represent the entire population from where the participants were recruited, no blinding for those responsible for assessing the outcomes, lack of adequate adjustment for the confounding variables in data analysis from which the findings were drawn, and inadequate sample size.

\section{Effectiveness of martial arts practice}

Effectiveness assessment of martial arts practice in overweight and obese subjects are presented as follows.

\section{$B M I$}

All studies of this systematic review assessed BMI (100\%), but only Chen and colleagues (16.66\%) [32] reported a significant difference between the intervention groups $\left(31.3 \mathrm{~kg} / \mathrm{m}^{2} ; \mathrm{SD}=4.2\right)$ and control groups $(32.8$ $\left.\mathrm{kg} / \mathrm{m}^{2} ; \mathrm{SD}=4.4\right)$ after the intervention period, with a lower BMI in the intervention group. However, four studies $(66.66 \%)$ [31, 32, 34, 35] reported a significant difference between the 'before and after' intervention period (Tables 3, 4).

Five studies (83.33\%) [30-34] in the meta-analysis assessed the outcome of martial arts interventions in relation to BMI (Fig. 2). The meta-analysis indicated a trend to reduce mean BMI for the groups that received martial arts interventions $-1.34 \mathrm{~kg} / \mathrm{m} 2 \quad(95 \%$ CI: $-2.72,0.05 ; \mathrm{I}^{2}=0 \%$ ), with $p=0.06$ and low level of heterogeneity.

\section{Body-fat percentage}

Three studies (50\%) assessed the percentage of body fat [30, 34, 35] (Tables 3, 4). However, only Dechamps and colleagues (16.66\%) [34] reported a significant difference between the intervention groups $(45.6 \% ; \mathrm{SD}=5.1)$ and control groups $(48.1 \% ; \mathrm{SD}=4.7)$ after the intervention period, with a lower percentage of body fat in the intervention group $(p<0.005)$. Nonetheless, two studies (33.33\%) [34, 35] reported a significant difference between the 'before and after' intervention period.

Three studies (50\%) [30, 34, 35] assessed the outcome of martial arts interventions in relation to body-fat percentage (Fig. 2). The examined meta-analysis did not reveal significant benefits from martial arts practice over control groups after the experiment period for percentage of body fat $-0.75 \%$ (95\% CI: $-5.58,4.08 ; \mathrm{I}^{2}=82 \%$ ), with $p=0.76$ and high level of heterogeneity.

\section{Circumferences}

Three studies $(50 \%)$ assessed waist circumference [30, 31, 35] (Tables 3, 4). However, only Tsang and colleagues (16.66\%) [30] reported a significant difference between the intervention groups $(100.2 \mathrm{~cm} ; \mathrm{SD}=19.3)$ and control groups $(103.6 \mathrm{~cm} ; \mathrm{SD}=17.1)$ after the intervention period, revealing a waist circumference increase in 
Table 2 Study quality assessment using the Downs \& Black tool

\begin{tabular}{|c|c|c|c|c|c|c|}
\hline Studies Item & Tsang et al. [30] & Beebe et al. [31] & Chen et al. [32] & Chyu et al. [33] & Dechamps et al. [34] & Katkowski et al. [35] \\
\hline D\&B: 1 & 1 & 1 & 1 & 1 & 1 & 1 \\
\hline D\&B: 2 & 1 & 1 & 1 & 1 & 1 & 1 \\
\hline D\&B: 3 & 1 & 1 & 1 & 1 & 1 & 1 \\
\hline D\&B: 4 & 1 & 1 & 1 & 1 & 1 & 1 \\
\hline D\&B: 5 & 0 & 0 & 0 & 0 & 0 & 0 \\
\hline D\&B: 6 & 1 & 1 & 1 & 1 & 1 & 1 \\
\hline D\&B: 7 & 1 & 1 & 1 & 1 & 1 & 1 \\
\hline D\&B: 8 & 1 & 0 & 1 & 1 & 1 & 0 \\
\hline D\&B: 9 & 1 & 0 & 0 & 1 & 1 & 0 \\
\hline D\&B: 10 & 1 & 1 & 1 & 1 & 1 & 1 \\
\hline D\&B: 11 & 0 & 0 & 0 & 0 & 0 & 0 \\
\hline D\&B: 12 & 0 & 0 & 0 & 0 & 0 & 0 \\
\hline D\&B: 13 & 1 & 0 & 0 & 0 & 0 & 0 \\
\hline D\&B: 14 & 1 & 0 & 0 & 0 & 1 & 0 \\
\hline D\&B: 15 & 1 & 0 & 0 & 1 & 1 & 0 \\
\hline D\&B: 16 & 1 & 1 & 1 & 1 & 1 & 1 \\
\hline D\&B: 17 & 1 & 0 & 1 & 1 & 1 & 0 \\
\hline D\&B: 18 & 1 & 1 & 1 & 1 & 1 & 1 \\
\hline D\&B: 19 & 0 & 0 & 0 & 1 & 0 & 1 \\
\hline D\&B: 20 & 1 & 1 & 1 & 1 & 1 & 1 \\
\hline D\&B: 21 & 1 & 0 & 1 & 1 & 1 & 1 \\
\hline D\&B: 22 & 1 & 0 & 1 & 1 & 1 & 1 \\
\hline D\&B: 23 & 1 & 1 & 1 & 1 & 1 & 1 \\
\hline D\&B: 24 & 1 & 0 & 0 & 1 & 1 & 0 \\
\hline D\&B: 25 & 0 & 0 & 0 & 0 & 1 & 0 \\
\hline D\&B: 26 & 1 & 0 & 0 & 1 & 1 & 0 \\
\hline D\&B: 27 & 0 & 0 & 3 & 0 & 0 & 1 \\
\hline Total & 21 & 11 & 18 & 20 & 21 & 15 \\
\hline
\end{tabular}

D\&B Downs \& Black item;

the intervention group (before $99.1 \mathrm{~cm} \pm 17.9$ and after $100.2 \mathrm{~cm} \pm 19.3$ ) and a decrease in the control group (before $106.5 \mathrm{~cm} \pm 16.7$ and after $103.6 \mathrm{~cm} \pm 17.1$ ). Two studies $(33.33 \%)$ [31, 35] reported a significant difference between the 'before and after' intervention period for both martial arts intervention and control groups.

Three studies (50\%) [30,31,35] assessed the outcomes of martial arts interventions in relation to waist circumference (Fig. 2). The meta-analysis showed no statistically significant difference in waist circumference between the martial arts intervention and control groups $\left(1.41 \mathrm{~cm} ; 95 \% \mathrm{CI}:-0.72,3.54 ; \mathrm{I}^{2}=0 \%\right)$, with $p=0.19$ and low level of heterogeneity.

\section{Discussion}

This systematic review evaluated the efficacy of martial arts training on the anthropometric and body composition parameters of overweight and obese subjects. It can be inferred that martial arts are a promising form of intervention for the treatment of overweight and obese subjects, since the meta-analysis showed a tendency to decrease BMI in those groups that received martial arts interventions (Fig. 2). However, there were no significant results from martial arts practice over control groups after the experiment period for waist circumference and percentage of body fat (Fig. 2). It should be pointed out, though, that most of the control groups received some intervention as well. Although mixed results have been reported, these findings were similar to those observed in other studies that analyzed the effects of aerobic exercise in postmenopausal overweight and obese women [36]. No systematic reviews were found assessing the effects of martial arts on the anthropometric and body composition parameters of overweight and 


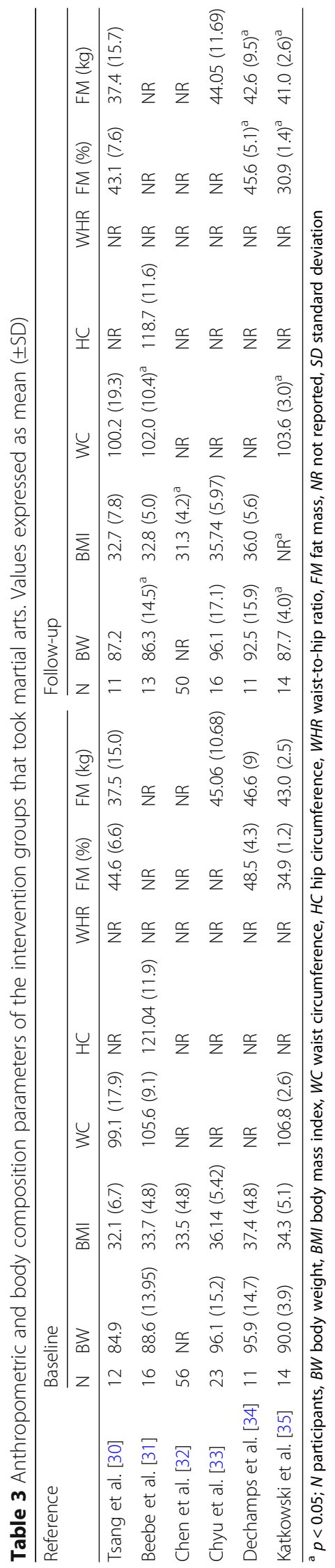


Table 4 Comparison of anthropometric and body composition parameters between treatment and control groups. Values expressed as mean (SD)

\begin{tabular}{|c|c|c|c|c|c|c|c|}
\hline \multirow[t]{2}{*}{ Study } & \multirow[t]{2}{*}{ Group } & \multicolumn{2}{|l|}{ BMI } & \multicolumn{2}{|c|}{ Waist circumference } & \multicolumn{2}{|c|}{$\%$ fat mass } \\
\hline & & Before & After & Before & After & Before & After \\
\hline \multirow[t]{2}{*}{ Tsang et al. [30] 2009} & Treatment & $32.1(6.7)$ & $32.7(7.8)$ & $99.1(17.9)$ & $100.2(19.3)$ & $44.6(6.6)$ & $43.1(7.6)$ \\
\hline & Control & $34.0(7.0)$ & $34.2(7.2)$ & $106.5(16.7)$ & $103.6(17.1)^{\mathrm{a}}$ & $47.7(5.3)$ & $47.4(5.6)$ \\
\hline \multirow[t]{2}{*}{ Beebe et al. [31] 2013} & Treatment & $33.7(4.8)$ & $32.8(5.0)$ & $105.6(9.1)$ & $102.0(10.4)^{b}$ & NR & NR \\
\hline & Control & $34.8(2.9)$ & $33.4(3.1)^{b}$ & $107.1(9.0)$ & $101.9(10.6)^{b}$ & NR & NR \\
\hline \multirow[t]{2}{*}{ Chen et al. [32] 2010} & Treatment & $33.5(4.8)$ & $31.3(4.2)^{a, b}$ & NR & NR & NR & NR \\
\hline & Control & $33.2(4.1)$ & $32.8(4.4)$ & NR & NR & NR & NR \\
\hline \multirow[t]{2}{*}{ Chyu et al. [33] 2013} & Treatment & $36.1(5.4)$ & $35.7(5.9)$ & NR & NR & NR & NR \\
\hline & Control & $35.6(7.1)$ & $36.2(7.7)$ & NR & NR & NR & NR \\
\hline \multirow[t]{2}{*}{ Dechamps et al. [34] 2009} & Treatment & $37.4(4.8)$ & $36.0(5.6)^{b}$ & NR & NR & $48.5(4.3)$ & $45.6(5.1)^{b}$ \\
\hline & Control & $38.5(7.3)$ & $39.3(8.2)$ & NR & NR & $50.3(7.0)$ & $48.1(4.7)$ \\
\hline \multirow[t]{2}{*}{ Katkowski et al. [35] 2013} & Treatment & $34.3(5.1)$ & $N R^{b}$ & $106.8(2.6)$ & $103.6(3.0)^{b}$ & $34.9(1.2)$ & $30.9(1.4)^{b}$ \\
\hline & Control & $34.8(2.9)$ & NR & $107.1(2.5)$ & $102.0(2.9)^{b}$ & $33.5(1.8)$ & $28.1(1.1)^{a, b}$ \\
\hline
\end{tabular}

${ }^{\mathrm{a}}$ Significant difference between treatment and control groups; ${ }^{\mathrm{b}}$ Significant difference between pre and post intervention; $p<0.05 ; B M I$ body mass index, NR not reported, $S D$ standard deviation

obese subjects. To our knowledge, this is the first study to investigate the topic. However, there is a review that investigated the effectiveness of martial arts for metabolic diseases [37] and another that investigated the Efficacy of Tai Chi and qigong for the prevention of stroke and stroke risk factors [38].

The results observed in this systematic review regarding the anthropometric and body composition parameters were incongruent, since some studies verified significant differences after the intervention period, whereas others did not. One factor that may have influenced the results was related to the age of the participants, since most of the studies [31-35] were conducted on older adults and elderly people. Only one study focused on adolescents [30], with several methodological problems. Another factor that might have influenced the results was the fact that most studies investigated solely Tai Chi style [31, 32, 34, 35]. Given that Tai Chi is a low-intensity physical exercise providing low-energy metabolism, with low energy-yielding metabolism, it does not favor beneficial changes in body composition [39].

A recent systematic review has investigated the benefits provided by the practice of hard martial arts in adults, and found they were safe and could provide several benefits, even for the elderly population [21]. However, these benefits could not be confirmed in the present review, as only two studies [30, 33] reported different martial arts interventions, and both had several methodological limitations. The study by Tsang and colleagues [30] was the only one included in this review that focused on adolescents as research subjects. The authors used Kung $\mathrm{Fu}$ as martial art intervention and Tai Chi as a placebo for the control group. That study had the longest duration ( 24 weeks), but the outcomes regarding changes in body composition and anthropometric parameters were not those expected, since the only difference between the intervention and control groups was the decrease in waist circumference in the control group that had Tai Chi intervention. This fact can be attributed to several factors related to the study, such as unintentional changes in the diet, number of participants (smaller than the required number by the sample size calculation - $\beta$ error), low participation rate in interventions $(<50 \%)$, and low intervention intensity due to the configuration of the training sessions [30].

Another study by Chyu and colleagues [33] adopted a differentiated intervention. The authors used various martial arts exercises as an intervention technique for women aged 30 years or older in the premenopausal period. This study also did not find positive outcomes in relation to changes in body composition, a fact that can be attributed to the short intervention period (12 weeks), lack of energy intake control, or the exercise intensity during the interventions, which were planned to be maintained between 50 and $70 \%$ of maximum heart rate [33]. This level of exercise intensity only allows modest changes in body composition, especially if administered alone. Thus, this was an inadequate treatment method for overweight or obese people [40].

The studies showing positive outcomes regarding changes in body composition used Tai Chi intervention followed by nutrition education and dietary intervention $[34,35]$. This was corroborated by Thorogood and colleagues [40] who found that moderate-intensity exercises accompanied by dietary and nutrition interventions can provide changes in body composition and be used as 


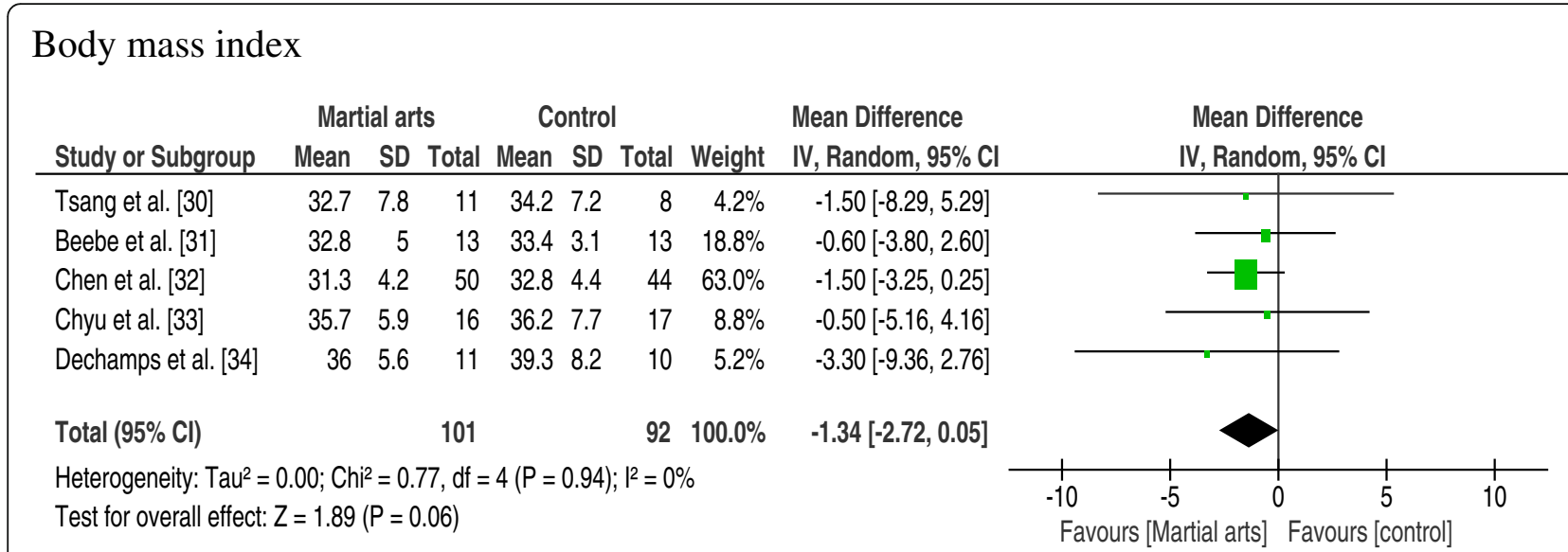

\section{Body-fat percentage}

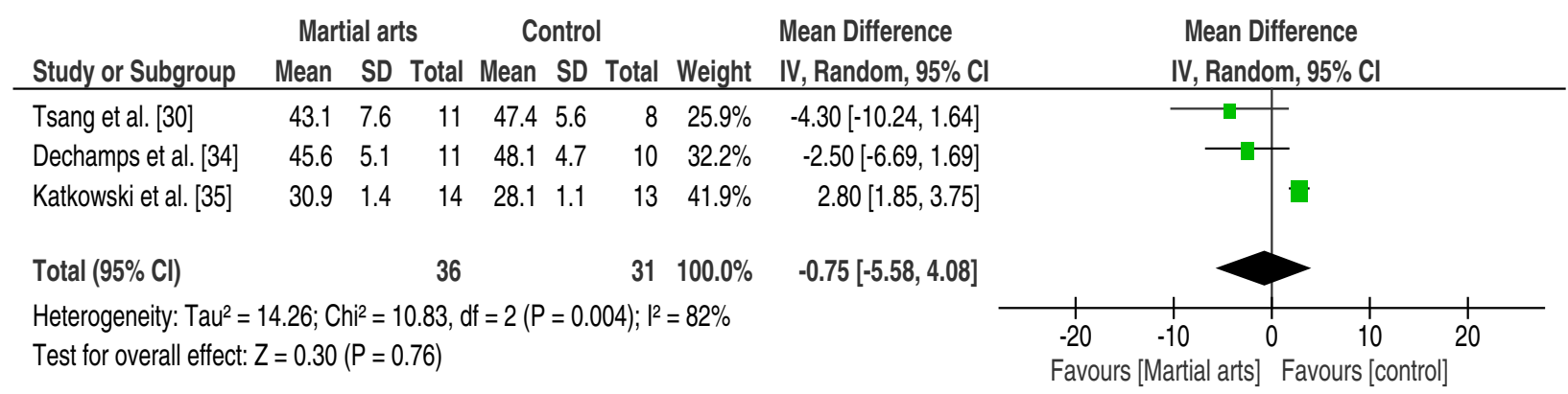

\section{Waist circumference}

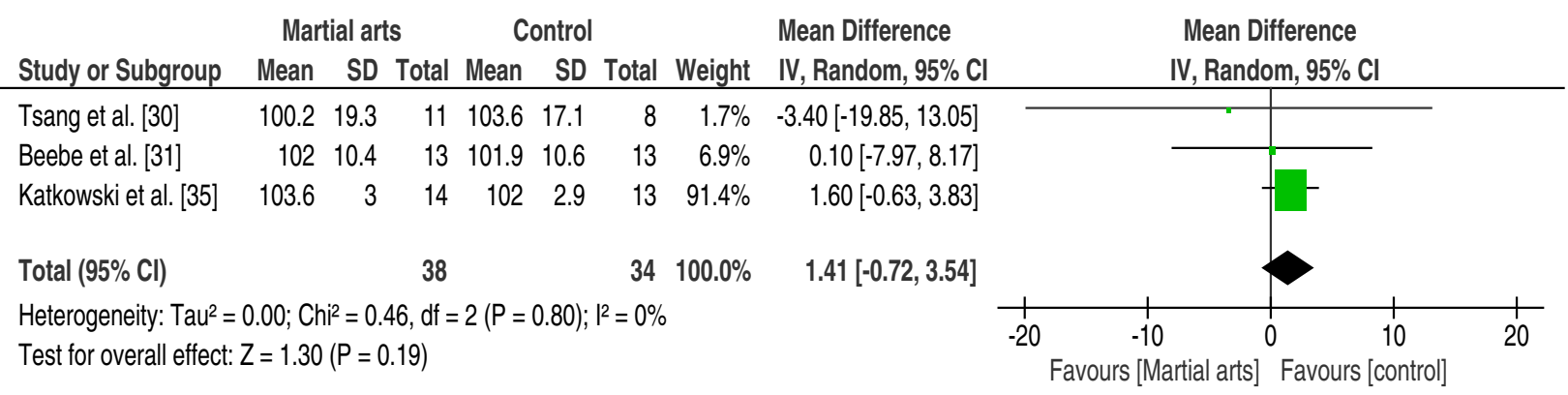

Fig. 2 Forest plot and effect sizes for martial arts compared with controls for body mass index, body-fat percentage and Waist circumference

treatment for overweight and obese subjects [40]. However, Chen and colleagues [32] employed only martial arts as an intervention technique and found a significant reduction in BMI. Nonetheless, that study enrolled a sample composed of people aged between 40 and 70 years, BMI of $\geq 30-35$ with type 2 diabetes mellitus. This specific population can respond differently to stimuli, since physical exercises are considered an important protection for people suffering from this disease [41]. Najafipour and colleagues [42] stated that regular aerobic exercise could improve body composition for people with type 2 diabetes mellitus, and Tai Chi could be considered a low-intensity aerobic exercise. In this population, regular exercises also provide a significant benefit in insulin sensitivity, which may persist beyond $72 \mathrm{~h}$ after the last exercise session [43].

\section{Limitations of this systematic review}

One of the challenges encountered in this review was the fact that all the studies adopted different methods, with different frequency and duration of training sessions, treatment lengths of time, and additional treatment interventions in the control groups, making it difficult to compare the studies. Another difficulty in 
pursuing the objectives of this review was the fact that most of the studies focused on Tai Chi as the martial art intervention, which is a low-intensity martial art.

This systematic review did not identify any major adverse events in any of the studies that composed it. However, $33.33 \%$ of the studies did not mention whether there were adverse events in their interventions, which could lead to misinterpretation of the safety of the interventions.

The results of the quality assessment of the studies that composed this review show that most studies obtained a medium-quality rating and none of them obtained a high score, which indicates more rigorous research studies are needed to determine the efficacy of the interventions (Table 2). Two of the review studies did not provide information about the sample size calculation [31, 34]. Two studies used sample sizes smaller than those required for the study $[30,33]$. None of the reviewed studies employed a randomized recruitment, and one of the studies did not report how the recruitment method was conducted [31].

Given that this was the first systematic review to examine the effects of martial arts interventions on overweight and obese subjects, all randomized clinical trials published up to January 2020 that met the inclusion criteria were encompassed in the review, regardless of their quality assessment.

\section{Implications for research}

Despite the limitations in this study, it should be highlighted that this was the first systematic review to assess martial arts exercise interventions for overweight and obese subjects.

This systematic review presented important outcomes from the studies that composed it. However, although the interventions have been relatively safe, it is important to conduct further research involving other types of martial arts, with more rigorous study designs, involving nutritional intervention with control of energy intake, assessment and control of daily activities, detailed description of the interventions and adverse events, larger sample sizes, and different research populations. These measures would allow us to obtain more conclusive results on the effects of martial arts as a treatment method for overweight and obese subjects. They would also help obtain results that can be generalized with confidence.

\section{Conclusion}

The scarcity, heterogeneity and significant methodological limitations of the available studies do not allow to conclude whether martial arts are effective in the anthropometric and body composition parameters of overweight and obese individuals. Martial arts can be an important tool for the treatment of overweight and obese people, as the results of this systematic review indicate that martial arts are probably safe and may offer benefits related to anthropometric and body composition parameters. However, the results are limited and should be used with caution. Because of this, further research is needed before we can generalize the findings regarding the efficacy of martial arts for maintaining healthy anthropometric parameters among overweight and obese subjects.

\section{Supplementary information}

Supplementary information accompanies this paper at https://doi.org/10. 1186/s12889-020-09340-x.

Additional file 1. Search strategy.

Additional file 2. Checklist for measuring study quality (Downs \& Black).

\section{Abbreviations}

BMI: Body mass index; MeSH: Medical Subject Headings; PRISMA: Preferred Reporting Items for Systematic reviews and Meta-Analyses; PROSPERO: International Prospective Register of Systematic Reviews; WHO: World Health Organization

\section{Acknowledgements \\ We are grateful to CAPES (Coordination for the Improvement of Higher Education Personnel) for granting a PhD scholarship to the first author.}

\section{Authors' contributions}

FS, MMMS, and DJT designed and developed the review study. FS and FNL were responsible for the online search, study selection, data extraction and assessing the methodological quality of the review studies. FS, FST, and DJT performed the data analyses. FS wrote the first draft of the manuscript. FS, MMMS, FNL, FST, and DJT participated in the critical review of the manuscript. All authors read and approved the final version of the manuscript.

\section{Funding}

This study had no funding.

\section{Availability of data and materials \\ Not applicable.}

Ethics approval and consent to participate

Not applicable.

Consent for publication

Not applicable.

\section{Competing interests}

The authors declare that they have no competing interests.

\section{Author details}

${ }^{1}$ Postgraduate Program in Health Sciences, University of Southern Santa Catarina, Avenida José Acácio Moreira, 787, Bairro Dehon, Tubarão, Santa Catarina Caixa Postal 370, Brazil. ${ }^{2}$ Degree in Biological Sciences, Leonardo da Vinci University Center, Capivari de Baixo, Santa Catarina, Brazil. ${ }^{3}$ Physical Education Degree, Leonardo da Vinci University Center, Capivari de Baixo, Santa Catarina, Brazil. ${ }^{4}$ Clinical Research Center, Hospital Nossa Senhora da Conceição, Tubarão, Santa Catarina, Brazil. 
Received: 1 March 2020 Accepted: 3 August 2020

Published online: 17 August 2020

\section{References}

1. World Health Organization. Geneva: obesity and overweight - fact sheet N³11; 2017. http://www.who.int/mediacentre/factsheets/fs311/en/; Accessed 17.12.03

2. World Obesity Federation. England and Wales: about obesity; 2015. http:// www.worldobesity.org/resources/aboutobesity/; Accessed 16.08.17.

3. World Health Organization Western Pacific Region, International Association for the Study of Obesity, International Obesity Task Force. Redefining obesity and its treatment; 2000. http://www.who.int/nutrition/publications/ obesity/09577082_1_1/en/; Accessed 17.12.03.

4. Donnelly JE, Blair SN, Jakicic JM, et al. American College of Sports Medicine position stand. Appropriate physical activity intervention strategies for weight loss and prevention of weight regain for adults. Med Sci Sports Exerc. 2009;41(2):459-71.

5. Verheggen RJ, Maessen MF, Green DJ, Hermus AR, Hopman MT, Thijssen $\mathrm{DH}$. A systematic review and meta-analysis on the effects of exercise training versus hypocaloric diet: distinct effects on body weight and visceral adipose tissue. Obes Rev. 2016;17(8):664-90.

6. Tchernof A, Després JP. Pathophysiology of human visceral obesity: an update. Physiol Rev. 2013;93(1):359-404.

7. Manna P, Jain SK. Obesity, oxidative stress, adipose tissue dysfunction, and the associated health risks: causes and therapeutic strategies. Metab Syndr Relat Disord. 2015;13(10):423-44.

8. Seabra A, Katzmarzyk P, Carvalho MJ, et al. Effects of 6-month soccer and traditional physical activity programmes on body composition, cardiometabolic risk factors, inflammatory, oxidative stress markers and cardiorespiratory fitness in obese boys. J Sports Sci. 2016;34(19):1822-9.

9. Boff RM, Liboni RP, Batista IP, Souza LH, Oliveira MD. Weight loss interventions for overweight and obese adolescents: a systematic review. Eat Weight Disord. 2017;22(2):211-29.

10. Fonseca-Junior SJ, Sá CGAB, Rodrigues PAF, Oliveira AJ, Fernandes-Filho J. Physical exercise and morbid obesity: a systematic review. Arq Bras Cir Dig. 2013;26(Suppl 1):67-73.

11. Blair EN, Manson JN, Paffenbarger RS. Epidemiologic methods in physical activity studies. New York: Oxford University Press; 2009.

12. Wareham NJ, Van Sluijs EM, Ekelund U. Physical activity and obesity prevention: a review of the current evidence. Proc Nutr Soc. 2005;64(2):22947.

13. Chin SH, Kahathuduwa CN, Binks M. Physical activity and obesity: what we know and what we need to know. Obes Rev. 2016;17(12):1226-44.

14. Marson EC, Delevatti RS, Prado AK, Netto N, Kruel LF. Effects of aerobic, resistance, and combined exercise training on insulin resistance markers in overweight or obese children and adolescents: a systematic review and meta-analysis. Prev Med. 2016;93:211-8.

15. Campos RM, Mello MT, Tock $L$, et al. Aerobic plus resistance training improves bone metabolism and inflammation in adolescents who are obese. J Strength Cond Res. 2014;28(3):758-66.

16. Dâmaso AR, Campos RMS, Caranti DA, et al. Aerobic plus resistance training was more effective in improving the visceral adiposity, metabolic profile and inflammatory markers than aerobic training in obese adolescents. J Sports Sci. 2014;32(15):1435-45.

17. Farah BQ, Ritti-Dias RM, Balagopal PB, Hill JO, Prado WL. Does exercise intensity affect blood pressure and heart rate in obese adolescents? A 6month multidisciplinary randomized intervention study. Pediatr Obes. 2014; 9(2):111-20.

18. Burke DT, Al-Adawi S, Lee YT, Audette J. Martial arts as sport and therapy. J Sports Med Phys Fitness. 2007;47(1):96-102.

19. Wang $X Q, P i$ YL, Chen PJ, et al. Traditional Chinese exercise for cardiovascular diseases: systematic review and meta-analysis of randomized controlled trials. J Am Heart Assoc. 2016;5(3):e002562.

20. Bu B, Haijun $H$, Yong $L$, Chaohui $Z$, Xiaoyuan $Y$, Singh MF. Effects of martial arts on health status: a systematic review. J Evid Based Med. 2010;3(4):20519.

21. Origua Rios S, Marks J, Estevan I, Barnett LM. Health benefits of hard martial arts in adults: a systematic review. J Sports Sci. 2017;36:1-9.

22. Sharpe PA, Blanck HM, Williams JE, Ainsworth BE, Conway JM. Use of complementary and alternative medicine for weight control in the United States. J Altern Complement Med. 2007;13(2):217-22.
23. Tsang TW, Kohn MR, Chow CM, Singh MF. Self-perception and attitude toward physical activity in overweight/obese adolescents: the "martial fitness" study. Res Sports Med. 2013;21(1):37-51.

24. Moher D, Liberati A, Tetzlaff J, Altman DG. Preferred reporting items for systematic reviews and meta-analyses: the PRISMA statement. Ann Intern Med. 2009;151(4):264-9.

25. Downs SH, Black N. The feasibility of creating a checklist for the assessment of the methodological quality both of randomised and non-randomised studies of health care interventions. J Epidemiol Community Health. 1998; 52(6):377-84.

26. Batacan Junior RB, Duncan MJ, Dalbo VJ, Tucker PS, Fenning AS. Effects of high-intensity interval training on cardiometabolic health: a systematic review and meta-analysis of intervention studies. Br J Sports Med. 2017; 51(6):494-503.

27. Higgins JPT, Thomas J, Chandler J, et al. Cochrane: Cochrane handbook for systematic reviews of interventions version 6.0 (updated July 2019); 2019. http://www.training.cochrane.org/handbook; Accessed 17.06.20.

28. Higgins JP, Thompson SG, Deeks JJ, et al. Measuring inconsistency in metaanalyses. BMJ. 2003;327:557-60.

29. Cohen J. Statistical power analysis for the behavioral sciences. Hillsdale: Lawrence Erlbaum Associates; 1988.

30. Tsang TW, Kohn MR, Chow CM, Singh MF. A randomised placebo-exercise controlled trial of kung Fu training for improvements in body composition in overweight/obese adolescents: the "martial fitness" study. J Sports Sci Med. 2009:8(1):97-106

31. Beebe N, Magnanti S, Katkowski L, et al. Effects of the addition of t'ai chi to a dietary weight loss program on lipoprotein atherogenicity in obese older women. J Altern Complement Med. 2013;19(9):759-66.

32. Chen SC, Ueng KC, Lee SH, Sun KT, Lee MC. Effect of t'ai chi exercise on biochemical profiles and oxidative stress indicators in obese patients with type 2 diabetes. J Altern Complement Med. 2010;16(11):1153-9.

33. Chyu MC, Zhang Y, Brismée JM, et al. Effects of martial arts exercise on body composition, serum biomarkers and quality of life in overweight/ obese premenopausal women: a pilot study. Clin Med Insights Womens Health. 2013;6:55-65.

34. Dechamps A, Gatta B, Bourdel-Marchasson I, Tabarin A, Roger P. Pilot study of a 10-week multidisciplinary tai chi intervention in sedentary obese women. Clin J Sport Med. 2009;19(1):49-53.

35. Katkowski LA, Benson MC, Magnanti S, Lofgren IE, Xu F, Delmonico MJ. Additive effect of tai chi during dietary weight loss on physical function and body composition in obese older women. J Aging Res Clin Pract. 2012; 2(1):78-85.

36. Baker A, Sirois-Leclerc $\mathrm{H}$, Tulloch $\mathrm{H}$. The impact of long-term physical activity interventions for overweight/obese postmenopausal women on adiposity indicators, physical capacity, and mental health outcomes: a systematic review. J Obes. 2016;2016:6169890.

37. Hamasaki H. Martial arts and metabolic diseases. Sports (Basel). 2016;4(2):E28.

38. Lauche $R$, Peng W, Ferguson $C$, et al. Efficacy of tai chi and qigong for the prevention of stroke and stroke risk factors: a systematic review with metaanalysis. Medicine (Baltimore). 2017;96(45):e8517.

39. Hui SS, Xie YJ, Woo J, Kwok TC. Practicing tai chi had lower energy metabolism than walking but similar health benefits in terms of aerobic fitness, resting energy expenditure, body composition and self-perceived physical health. Complement Ther Med. 2016;27:43-50.

40. Thorogood A, Mottillo S, Shimony A, et al. Isolated aerobic exercise and weight loss: a systematic review and meta-analysis of randomized controlled trials. Am J Med. 2011;124(8):747-55.

41. Boyer WR, Churilla JR, Ehrlich SF, Crouter SE, Hornbuckle LM, Fitzhugh EC. Protective role of physical activity on type 2 diabetes: analysis of effect modification by race-ethnicity. J Diabetes. 2017;10(2):166.

42. Najafipour F, Mobasseri M, Yavari A, et al. Effect of regular exercise training on changes in $\mathrm{HbA1c}$, BMI and $\mathrm{VO} 2$ max among patients with type 2 diabetes mellitus: an 8-year trial. BMJ Open Diabetes Res Care. 2017;5(1): e000414.

43. Way KL, Hackett DA, Baker MK, Johnson NA. The effect of regular exercise on insulin sensitivity in type 2 diabetes mellitus: a systematic review and meta-analysis. Diabetes Metab J. 2016;40(4):253-71.

\section{Publisher's Note}

Springer Nature remains neutral with regard to jurisdictional claims in published maps and institutional affiliations. 